\title{
Task Control with Remote Center of Motion Constraint for Minimally Invasive Robotic Surgery
}

\author{
Nastaran Aghakhani Milad Geravand Navid Shahriari Marilena Vendittelli Giuseppe Oriolo
}

\begin{abstract}
Minimally invasive surgery assisted by robots is characterized by the restriction of feasible motions of the manipulator link constrained to move through the entry port to the patient's body. In particular, the link is only allowed to translate along its axis and rotate about the entry point. This requires constraining the manipulator motion with respect to a point known as Remote Center of Motion (RCM). The achievement of any surgical task inside the patient's body must take into account this constraint. In this paper we provide a new, general characterization of the RCM constraint useful for task control in the minimally invasive robotic surgery context. To show the effectiveness of our formalization, we consider first a visual task for a manipulator with 6 degrees of freedom holding an endoscopic camera and derive the kinematic control law allowing to achieve the visual task while satisfying the RCM constraint. An example of application of the proposed kinematic modeling to a motion planning problem for a 9 degrees of freedom manipulator with assigned path for the surgical tool is then proposed to illustrate the generality of the approach.
\end{abstract}

\section{INTRODUCTION}

The operating modality of Minimally Invasive Surgery (MIS) consists in introducing the surgical tools inside the patient's body through small incisions and in guiding them from the outside of the body. In addition to the tools necessary to perform the surgery, a camera is introduced into the body to provide the surgeon with the view of the surgery site. Though highly advantageous for the patients, these operative conditions constrain the manipulation and are less natural and more tiring for the surgeon with respect to open surgery.

The introduction of robots in the operative room is aimed at recovering the conditions of the open surgery while providing an important support in terms of accuracy and comfort for the surgeon during the accomplishment of the surgical task. Robotic arms are used for moving the endoscopic camera only or the camera and the surgical tools. Whatever the setup of the robot-assisted surgery system is, the used manipulators must posses a special kinematics obtained either through mechanical design or by appropriate control of the manipulator joints. More specifically, the robot link constrained to move through the incision point (usually the link holding the surgical tool), can only translate along its axis and rotate about the incision point. The manipulator motion is then constrained with respect to a point on the link axis known as Remote Center of Motion (RCM).

Many different kinds of mechanically constrained RCM manipulators have been proposed in the literature (see,

The authors are with the Dipartimento di Ingegneria Informatica, Automatica e Gestionale, Università di Roma La Sapienza, via Ariosto 25, 00185, Roma, Italy. E-mail: \{oriolo,vendittelli\}@dis.uniroma1.it. This work is supported by the EU FP7 ICT-287513 SAPHARI project. e.g., [1], [2], [3] and the included references) as the mechanical lock of RCM is generally considered safer than the constrained control of manipulators [2]. However, obtaining a programmable RCM by constraining the motion of a serial robotic arm represents a more flexible and space-efficient solution also allowing the use of the same manipulator both for open and for minimally invasive surgery [4]. This solution has been considered in several works, like, e.g., [5], [6], [7], [8], [9], [10], [11]. In these papers, the RCM constraint satisfaction is guaranteed through the presence of passive joints and appropriate control law [7], or by extending the surgical task with the RCM constraint [8], or by real-time optimization methods [9], [10], [11], [12].

The main contribution of the paper is to propose a formalization of the RCM constraint that explicitly model the translational motion along the link axis and therefore allows a direct control of a variable representing the link penetration into the patient's body. This is a distinguishing feature with respect to [13] which is strictly related to the present work. In addition, with the proposed formalism only the position of the tool insertion point is required instead of the equation of the plane tangent to the body at the trocar position usually needed by other methods, including [13].

The constraint is derived considering general serial manipulators. Extension of any given task with the proposed RCM constraint formulation allows easy derivation of a kinematic control law. In particular, we consider in this paper a visual task for a 6 degrees of freedom manipulator subject to RCM constraint and a motion planning problem for a manipulator with 9 degrees of freedom that must follow an assigned path with the surgical tool.

This paper is organized as follows, in Sect. II the considered constraint is described and mathematically formulated. In Sect. III a kinematic control scheme is derived. Application of the proposed approach to the case of a visual and a motion planning task are proposed respectively in Sect. IV and Sect. V. The final section proposes concluding remarks.

\section{THE RCM CONSTRAINT}

Minimally invasive surgery (MIS) requires the introduction of an endoscopic camera and one or more surgical tools into the patient's body through small incisions. The execution of any task inside the body is therefore conditioned by the passage of the surgical instruments through the incision point. This imposes a constraint on the manipulator motion known as Remote Center of Motion (RCM) constraint. We propose a formalization of the RCM constraint that can be used in combination with other tasks for control design. 


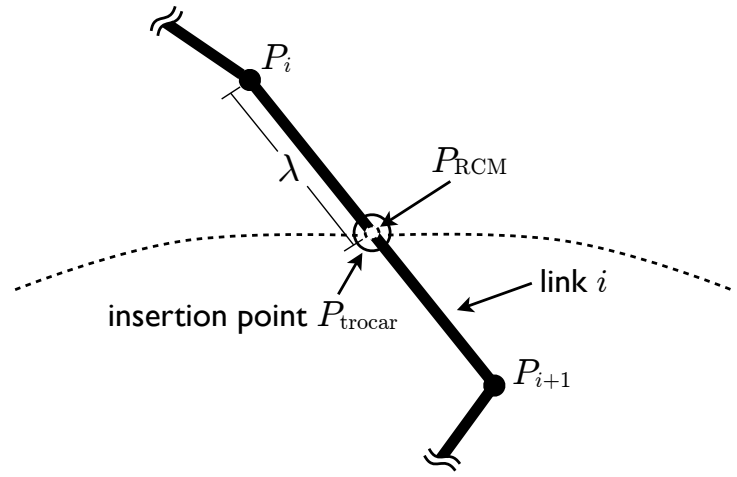

Fig. 1. The RCM is a variable point on a given robot link at the entry point of the patient's body.

Consider a serial manipulator with $n$ joints of which $i$ are possibly outside the body and $n-i$ inside. With reference to Fig. 1, assume that a specific surgical task requires that the manipulator $i$-th link is constrained to pass, while moving, through a fixed point $P_{\text {trocar }} \in \boldsymbol{R}^{3}$, the point where the surgical tool is inserted into the body through a trocar. The point on the link axis instantaneously coincident with $P_{\text {trocar }}$ and denoted by $P_{\mathrm{RCM}}$ represents an RCM for the manipulator and, in principle, it can be located anywhere along the segment with endpoints $P_{i}, P_{i+1}$ that represent the origin of the Denavit-Hartenberg frames associated to the manipulator joints $i$ and $i+1$ respectively.

Let $\boldsymbol{q}=\left(q_{1} \ldots q_{n}\right)^{T}$ be the vector of joint variables, and denote with $\boldsymbol{p}_{i}(\boldsymbol{q}(t)), \boldsymbol{p}_{i+1}(\boldsymbol{q}(t))$ the cartesian coordinates of $P_{i}$ and $P_{i+1}$, respectively, in the manipulator base frame ${ }^{1}$. The RCM point $P_{\mathrm{RCM}}$, with coordinates $\boldsymbol{p}_{\mathrm{RCM}}(\boldsymbol{q}(t))$, belongs to the segment with endpoints $P_{i}, P_{i+1}$ and its position is allowed to change with time along the segment due to the manipulator motion:

$$
\begin{array}{r}
\boldsymbol{p}_{\mathrm{RCM}}(\boldsymbol{q}(t))=\boldsymbol{p}_{i}(\boldsymbol{q}(t))+\lambda(t)\left(\boldsymbol{p}_{i+1}(\boldsymbol{q}(t))-\boldsymbol{p}_{i}(\boldsymbol{q}(t))\right), \\
0 \leq \lambda(t) \leq 1 .
\end{array}
$$

Differentiating eq. (1) with respect to time we obtain

$$
\dot{\boldsymbol{p}}_{\mathrm{RCM}}=\dot{\boldsymbol{p}}_{i}+\dot{\lambda}\left(\boldsymbol{p}_{i+1}-\boldsymbol{p}_{i}\right)+\lambda\left(\dot{\boldsymbol{p}}_{i+1}-\dot{\boldsymbol{p}}_{i}\right)
$$

where we have dropped the dependencies for brevity. Expressing the velocity $\dot{\boldsymbol{p}}_{i}, \dot{\boldsymbol{p}}_{i+1}$ of points $P_{i}$ and $P_{i+1}$ through the associated jacobians, respectively $\boldsymbol{J}_{i}$ and $\boldsymbol{J}_{i+1}$, gives

$$
\dot{\boldsymbol{p}}_{\mathrm{RCM}}=\boldsymbol{J}_{i} \dot{\boldsymbol{q}}+\dot{\lambda}\left(\boldsymbol{p}_{i+1}-\boldsymbol{p}_{i}\right)+\lambda\left(\boldsymbol{J}_{i+1} \dot{\boldsymbol{q}}-\boldsymbol{J}_{i} \dot{\boldsymbol{q}}\right) .
$$

Rewriting (2) in matrix notation yields

$$
\dot{\boldsymbol{p}}_{\mathrm{RCM}}=\left(\begin{array}{c}
\boldsymbol{J}_{i}+\lambda\left(\boldsymbol{J}_{i+1}-\boldsymbol{J}_{i}\right) \\
\boldsymbol{p}_{i+1}-\boldsymbol{p}_{i}
\end{array}\right)^{T}\left(\begin{array}{c}
\dot{\boldsymbol{q}} \\
\dot{\lambda}
\end{array}\right)
$$

or, in compact form,

$$
\dot{\boldsymbol{p}}_{\mathrm{RCM}}=\boldsymbol{J}_{\mathrm{RCM}}(\boldsymbol{q}, \lambda)\left(\begin{array}{c}
\dot{\boldsymbol{q}} \\
\dot{\lambda}
\end{array}\right) .
$$

\footnotetext{
${ }^{1}$ Obviously, if $i<n$ joints are outside the body, the position of these points depend on the value of the joints $q_{1}$ to $q_{i}$ only. The formalization that we derive here holds in the general case.
}

The trocar condition requires that the cartesian coordinates of $P_{\mathrm{RCM}}$ must be constantly equal to the the insertion point, i.e., $\boldsymbol{p}_{\mathrm{RCM}} \equiv \boldsymbol{p}_{\text {trocar }}$. Therefore, their derivative with respect to time must be identically equal to zero:

$$
\dot{\boldsymbol{p}}_{\mathrm{RCM}}=\boldsymbol{J}_{\mathrm{RCM}}(\boldsymbol{q}, \lambda)\left(\begin{array}{c}
\dot{\boldsymbol{q}} \\
\dot{\lambda}
\end{array}\right)=\mathbf{0} .
$$

Note that in deriving the RCM constraint we have followed the same approach as [13]. In that work, however, the derivative of $\lambda$ is not included in the computation of $\boldsymbol{J}_{\mathrm{RCM}}$. In many surgical tasks, however, variation of $\lambda$ with respect to time could be required and its time derivative should be considered in the computation of $\boldsymbol{J}_{\mathrm{RCM}}$. In addition, in [13] the equation of the plane tangent to the body is necessary to compute $\boldsymbol{J}_{\mathrm{RCM}}$. This assumption is however critical in practical applications since this equation should be determined through a registration procedure involving modeling of the body surface which is prone to errors and inevitably requires approximations. RCM constraint described by (3-4) does not make use of the equation of the plane tangent to the body surface at the entry point of the surgical tool.

It is also worth noting that although eq. (4) represents a constraint of dimension 3 , the presence of $\dot{\lambda}$, explicitly modeling variation of the penetration, increases the degrees of freedom by 1 . Hence, the number of residual degrees of freedom available for achieving additional tasks remains unchanged with respect to the classical formulation of this constraint, i.e., there is a loss of 2 dof due to the RCM constraint or, equivalently, the RCM originates a constraint of dimension 2.

Finally, note that the direct control on $\lambda$ allowed by the proposed formalism, is convenient to guarantee that the penetration remains within a prescribed interval and useful for controlling the manipulator during the insertion phase.

\section{Control Design}

Consider a robot task expressed in coordinates $t$ which takes values in an $n_{t}$-dimensional task space $\mathcal{T}$. The task coordinates are related to the joint variables $\boldsymbol{q}$ through the kinematic map

$$
\boldsymbol{t}=\boldsymbol{f}(\boldsymbol{q}) .
$$

At the differential level, it is

$$
\dot{\boldsymbol{t}}=\boldsymbol{J}_{t}(\boldsymbol{q}) \boldsymbol{q}
$$

where $\boldsymbol{J}_{t}(\boldsymbol{q})$ is the $n_{t} \times n$ task Jacobian mapping joint velocities to task space velocities. Assuming that the task must be achieved while satisfying an RCM constraint, the manipulator must have $n \geq n_{t}+2$ degrees of freedom to allow simultaneous task execution and RCM constraint satisfaction, since the RCM constraint has dimension 2.

Using the RCM constraint formulation (4) and task kinematics (5) we define the kinematics of the extended task

$$
\dot{\boldsymbol{t}}_{\mathrm{EXT}}=\left(\begin{array}{l}
\dot{\boldsymbol{t}} \\
\mathbf{0}_{3 \times 1}
\end{array}\right)=\left(\begin{array}{ll}
\boldsymbol{J}_{t} & \mathbf{0}_{n_{t} \times 1} \\
& \boldsymbol{J}_{\mathrm{RCM}}
\end{array}\right)\left(\begin{array}{l}
\dot{\boldsymbol{q}} \\
\dot{\lambda}
\end{array}\right)
$$

where we have dropped the arguments of the jacobians. 
Assigned a desired extended task $\boldsymbol{t}_{d, \mathrm{EXT}}=\left(\begin{array}{ll}\boldsymbol{t}_{d} & \boldsymbol{p}_{\text {trocar }}\end{array}\right)^{T}$, and defined the task error

$$
\boldsymbol{e}_{t}=\left(\begin{array}{c}
\boldsymbol{t}_{d}-\boldsymbol{t} \\
\boldsymbol{p}_{\text {trocar }}-\boldsymbol{p}_{\mathrm{RCM}}
\end{array}\right),
$$

in the hypothesis that the manipulator is redundant with respect to the extended task, i.e., $n>n_{t}+2$ the following kinematic control

$$
\left(\begin{array}{c}
\dot{\boldsymbol{q}} \\
\dot{\lambda}
\end{array}\right)=\boldsymbol{J}^{\#}\left(\begin{array}{cc}
\boldsymbol{K}_{t} & \boldsymbol{0}_{n_{t} \times 3} \\
\boldsymbol{0}_{3 \times n_{t}} & \boldsymbol{K}_{\mathrm{RCM}}
\end{array}\right) \boldsymbol{e}_{t}+\left(\boldsymbol{I}-\boldsymbol{J}^{\#} \boldsymbol{J}\right) \boldsymbol{w},
$$

with $\boldsymbol{K}_{t}$ and $\boldsymbol{K}_{\mathrm{RCM}}$ positive definite diagonal matrices of dimension $n_{t} \times n_{t}$ and $3 \times 3$ respectively, and $\boldsymbol{J}^{\#}$ the pseudoinverse of $\boldsymbol{J}$, the Jacobian of the extended task, guarantees decoupled exponential convergence of the task to the desired value.

The vector $\boldsymbol{w}$ in eq. (7) is a residual input, projected in the null space of $\boldsymbol{J}$, which is useful to satisfy additional tasks like joint limits avoidance and, in particular, limits on the variable $\lambda$ modeling the penetration. Assuming that, for a given application, it is convenient to keep $\lambda$ as close as possible to a fixed value $\lambda_{0}$ we can choose as null-space vector the gradient of the function $d=\frac{1}{2}\left(\lambda-\lambda_{0}\right)^{2}$, i.e., $\boldsymbol{w}=-\left(\nabla_{(\boldsymbol{q}, \lambda)} d\right)^{T}=\left(\begin{array}{llll}0 & \ldots & 0 & \lambda_{0}-\lambda\end{array}\right)^{T}$.

An alternative way to exploit redundancy is the optimization of some index measuring the compatibility of a given posture with respect to the task of interest. In the next section we will illustrate both uses in the case of a visual task.

Note that, the kinematic control (7) requires to know only the position of the insertion point $P_{\text {trocar }}$ with respect to the manipulator base frame. Assuming that the manipulator is accurately calibrated, this position can be obtained by a simple registration procedure consisting, for example, in positioning manually the manipulator tool tip in the intraoperative phase or by using an optically tracked probe. Of course, this will provide also the initial value of $\lambda$.

In the following developments, we assume that the positioning of the robot with respect to the insertion point has been determined by an optimization procedure that should guarantee that algorithmic singularities could not be encountered in normal procedure execution.

\section{VISUAL TASK}

Robotically assisted MIS setup includes at least one endoscopic vision system composed by a monocular camera with light emission. The camera is usually mounted on the end effector of a manipulator and can be positioned manually, by voice control or using visual information. In this last case, visual servoing control with eye-in-hand configuration is often used to localize and track instruments inside the body (in most applications the abdomen cavity) or to keep a desired target at the center of the image plane rejecting also periodical motions like breathing or heart beating [14]. If a geometric model of the object to track is not available, IBVS represents a suitable choice for its robustness with respect to camera calibration errors.
Different types of vision systems capable of tracking and guiding a laparoscopic camera to a desired view or the surgical tools to a pose of interest have been developed in the past years as a robotic support to surgery (examples include [15], [16], [17], [18]). Work on visual servoing in the MIS context is often focused on the challenging problem of capturing the visual information necessary to determine the reference velocities to be sent to the manipulator controller [19].

Few works consider the extension of the visual task with the RCM constraint for control design and in most cases the emphasis is on control rather than on the vision related issues mentioned above. The application of the proposed kinematic formalization of the RCM constraint to a visual servoing problem is in this vein. There is no claim of proposing a welldesigned surgical task. The example has been constructed to show the properties of the proposed formulation and its potentialities within a robotically assisted MIS context.

Very closely related to the example considered in this paper are [17], [18] in which, however, the mathematical characterization of the RCM constraint is different with respect to the aspects discussed above, and in particular does not allow direct control on the penetration variable.

With the aim of recalling here the fundamental equations of an IBVS scheme, consider a target of interest in the field of view of a camera positioned at the end effector of a manipulator. Denote with $\boldsymbol{f}$ the $n_{f}$-dimensional vector of visual features on the image plane associated to the target and $Z$ the target depth. The velocity of the features in the image plane is related to the velocity of the camera $\left(\boldsymbol{v}_{c} \boldsymbol{\omega}_{c}\right)^{T}$, expressed in its frame, through the so-called interaction matrix $\boldsymbol{J}_{i}(f, Z)[20]$ :

$$
\dot{\boldsymbol{f}}=\boldsymbol{J}_{i}(\boldsymbol{f}, Z)\left(\begin{array}{c}
\boldsymbol{v}_{c} \\
\boldsymbol{\omega}_{c}
\end{array}\right) .
$$

The differential mapping between $f$ and the manipulator joint velocities can then be obtained by relating the camera velocity $\left(\boldsymbol{v}_{c} \boldsymbol{\omega}_{c}\right)^{T}$ to the manipulator joint velocities through the camera jacobian $\boldsymbol{J}_{c}(\boldsymbol{q})$ :

$$
\dot{\boldsymbol{f}}=\boldsymbol{J}_{i}(\boldsymbol{f}, Z) \boldsymbol{J}_{c}(\boldsymbol{q}) \dot{\boldsymbol{q}}=\boldsymbol{J}_{v}(\boldsymbol{f}, \boldsymbol{q}, Z) \dot{\boldsymbol{q}} .
$$

The visual task consists in regulating the coordinates of the point feature to the desired value $f_{d}$.

Note that evaluation of matrix $\boldsymbol{J}_{i}(\boldsymbol{f}, Z)$ requires knowledge of the feature depth $Z$ that we have assumed to be exactly known in the proposed simulations. In application to real systems, it could be estimated by using the nonlinear observer proposed in [21]. Feature depth estimation in MIS context is however beyond the scope of the present paper. Note also that the proposed visual servoing scheme can be used to track the surgical tools (eye-to-end configuration) and that in this case the depth of the feature points on the tools can be obtained by system calibration as in [18].

The dependence of the features Jacobian $\boldsymbol{J}_{v}$ on the features value and depth does not affect the formal expression of the extended task which takes exactly the same form as (6) with $\boldsymbol{t}=\boldsymbol{f}, \boldsymbol{J}_{v}$ in place of $\boldsymbol{J}_{t}$ and $n_{t}=n_{v}$. 
The task error in this case is

$$
\boldsymbol{e}_{t}=\left(\begin{array}{c}
\boldsymbol{f}_{d}-\boldsymbol{f} \\
\boldsymbol{p}_{\text {trocar }}-\boldsymbol{p}_{\mathrm{RCM}}
\end{array}\right)
$$

and the kinematic control law (7) reads exactly the same with appropriate matrix dimensions and definition of $\boldsymbol{J}$.

Considering that at the beginning of the procedure the camera is positioned at the insertion point (or the link is already inserted in the body), the control law (7) guarantees that the RCM error is kept equal to zero while a real regulation for the visual features is achieved. Numerical error correction as well as tracking of small displacements of the trocar due to unwanted patient motion is possible thanks to the cartesian correction term, provided that the trocar position is on-line estimated or tracked with an external device.

\section{A. Simulation results}

In this section we present the simulation results obtained by application of the proposed control scheme to a model of the KUKA KR5 Sixx R650 manipulator in the Webots environment. We have modified the length of the last link and added a camera at the tip. Figure 2 shows a sequence of snapshots from the first simulation.

The considered simulation setup has been constructed to put in evidence the characteristic of the proposed method. It represents an abstraction of the automatic camera positioning scenario in the MIS context. The transparent surface represents the body at the entry point and the trocar is represented by the small (yellow) sphere. In all the proposed simulations we assume that the camera is initially positioned at the insertion point. This implies that the initial value of the penetration variable is equal to zero ${ }^{2}$. The visual target is represented by the black disk in the bottom right corner of each snapshot.

In the first two simulations the visual task is to regulate the position of the observed target center to the center of the image plane, i.e, the visual feature is a point and $n_{f}=2$. The degree of redundancy with respect to the extended task $\boldsymbol{t}_{\mathrm{EXT}}$ is therefore equal to 2 . The task gains in both cases are $\boldsymbol{K}_{t}=\operatorname{diag}\{0.3,0.3\}$ and $\boldsymbol{K}_{\mathrm{RCM}}=\operatorname{diag}\{2.5,2.5,2.5\}$.

Simulation 1 has been run with $\boldsymbol{w}=\mathbf{0}$, hence there is no control on the penetration of the last link that can, therefore, assume any value. Figure 2 shows, in clockwise direction starting from the top left snapshot, a case in which the link moves away from the surface through the insertion point while pointing to the trocar, the visual target is occluded and the task is not completed. See the video attachment to this paper for a movie clip of this and all other simulations.

To show the effectiveness of our approach, in Simulation 2 we added the null-space vector $\boldsymbol{w}=\left(0,0,0,0,0,0, \lambda_{0}-\lambda\right)$ with $\lambda_{0}=0.35$. Snapshots from this second simulation are reported in Fig. 3. Side by side on each snapshot are shown the manipulator configuration (left) and the current

\footnotetext{
${ }^{2}$ It is worth noting that initialization of $\lambda$ to zero is not necessary for convergence of the visual task. An unmatched value of $\lambda$ would result in any case in exponential convergence of the visual features to the desired value. Since the manipulator is redundant with respect to the considered visual tasks, this would imply only different joint trajectories.
}

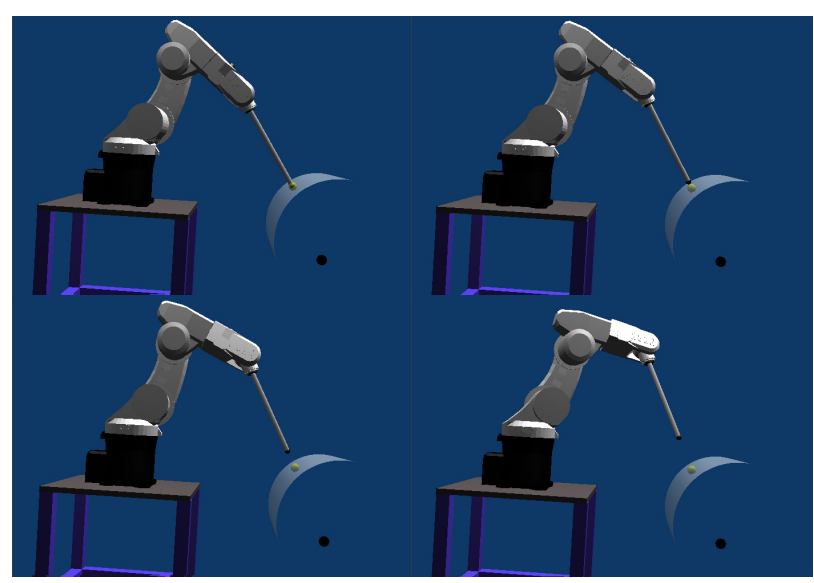

Fig. 2. Simulation1: task execution without control on the penetration.

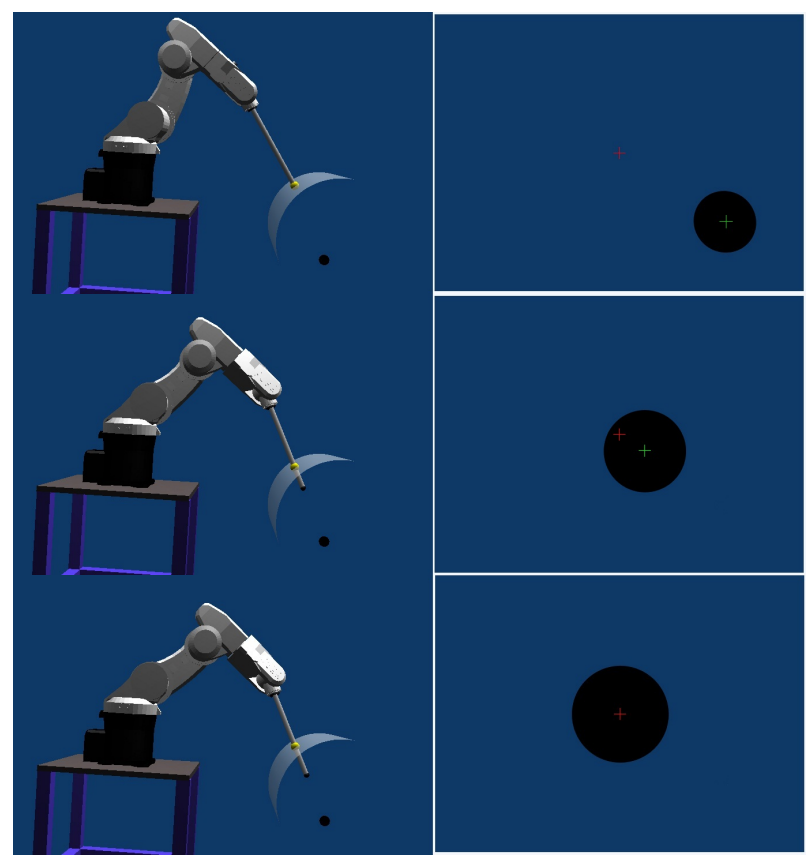

Fig. 3. Simulation 2: task execution with control on the penetration.

view of the camera (right). The manipulator moves the camera to regulate the position of the center of the black disk (the image tartget) satisfying the RCM constraint and increasing the penetration value. The last snapshot shows the successful accomplishment of the visual task with a non-zero penetration value. Exponential convergence of the visual error is shown in Fig. 4, while Fig. 5 reports the joint velocities. In this case, as shown in Fig. $6, \lambda$ stays close to the desired value while the task is achieved.

To study the influence of the RCM constraint on the visual task manipulability we have considered the velocity transmission ratio defined in [22]

$$
\beta=\left(\boldsymbol{u}^{T}\left(\boldsymbol{J} \boldsymbol{J}^{T}\right)^{-1} \boldsymbol{u}\right)^{-1 / 2} .
$$




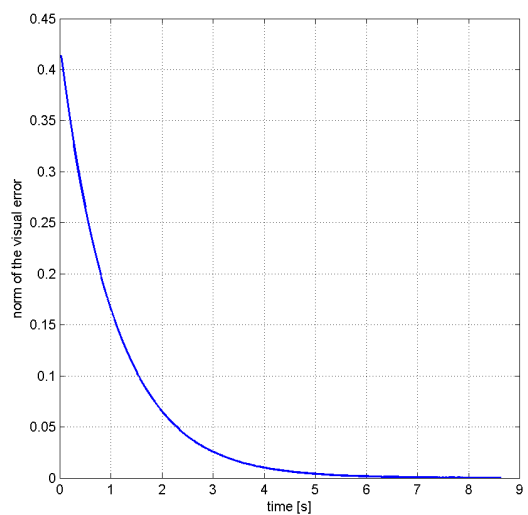

Fig. 4. Simulation 2: norm of the visual error.

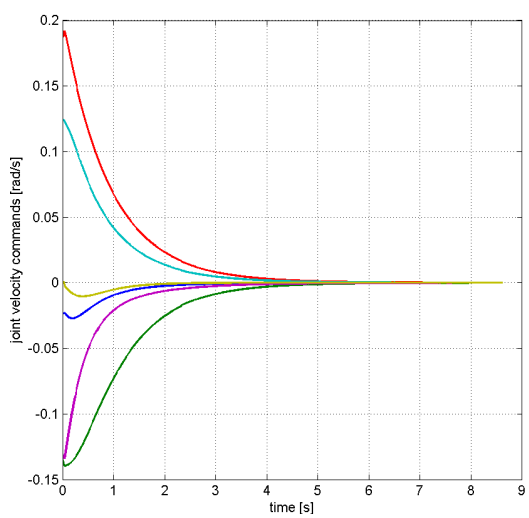

Fig. 5. Simulation 2: joint velocities.

This manipulability index is equal to the distance from the center to the surface of the manipulability ellipsoid along the direction of $\boldsymbol{u}$ indicating the versor of the task. Considering that the convergence is linear and decoupled in the visual task space and that the RCM is fixed, the unit vector $\boldsymbol{u}$ can be computed as $\boldsymbol{u}=\frac{\tilde{\boldsymbol{u}}}{|\tilde{\boldsymbol{u}}|}$, where $\tilde{\boldsymbol{u}}=\left(\begin{array}{ll}\boldsymbol{f} & \mathbf{0}\end{array}\right)^{\mathbf{T}}$ is the current task value and $|\tilde{\boldsymbol{u}}|$ denotes the euclidean norm of $\tilde{\boldsymbol{u}}$.

Figure 7 reports a comparative plot of the value assumed by the index $\beta$ in achieving the visual task with and without the RCM constraint. The simulation results suggest that this index is very sensitive to the restriction of the manipulability introduced by the RCM and, therefore, its maximization is expected to improve the controller performance. Although we have not pursued this possibility in the present paper it could be the subject of further work.

A third simulation was run to show the performance of the proposed control scheme when the visual task is to regulate the center of the observed target to the center of the image plane and its area to a desired value. In this case $n_{f}=3$ and the degree of redundancy with respect to the extended task $\boldsymbol{t}_{\mathrm{EXT}}$ is equal to 1 . The task gains were chosen as $\boldsymbol{K}_{t}=$ $\operatorname{diag}\{0.3,0.3,0.15\}$ and $\boldsymbol{K}_{\mathrm{RCM}}=\operatorname{diag}\{2.5,2.5,2.5\}$. As in the previous simulations, the visual errors converge

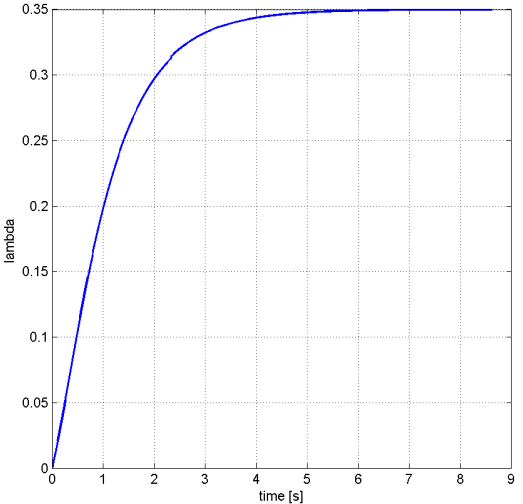

Fig. 6. Simulation 2: $\lambda$ approaches the desired value.

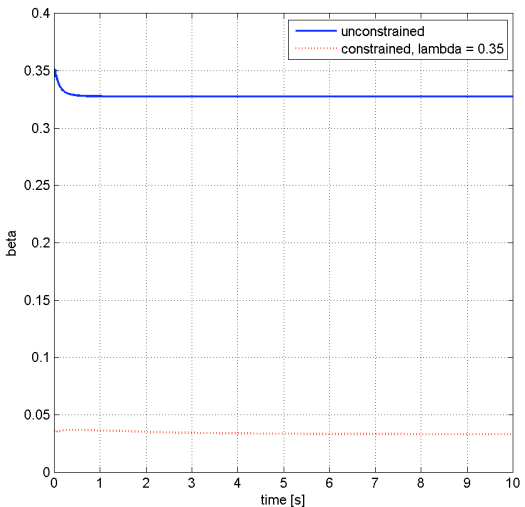

Fig. 7. Compatibility index in the constrained (dotted, red line) and unconstrained (continuous, blue line) visual task execution.

exponentially while the RCM constraint remains satisfied. Plots are not reported for lack of space but a movie clip of the simulation is included in the video attached to the paper.

\section{PATH FOLLOWING TASK}

In this section we briefly illustrate a motion planning example that proves the generality of the RCM modeling and task control approach. Motion planning methodologies could represent a powerful tool in the future in the preoperative planning phase and in guaranteeing safety of operations and in the automatization of routine procedures like suturing.

The reported motion planning experiment that is a preliminary result on ongoing research on motion planning in surgical robotics and represents an abstract task. Figure 8 illustrates the scenario: a manipulator with 9 degrees of freedom ( 2 of which are inside the body during surgery), representing a model of the DLR MIRO robot, must follow a cartesian path assigned to the surgical tool while satisfying the RCM constraint and avoiding the obstacles inside (organs) and outside (medical tools and furnitures) the patient body as well as self-collisions. The extended task has the form (6) with $t$ representing the cartesian coordinates of the path, $\boldsymbol{J}_{t}$ the task Jacobian with $n_{t}=3$. The degree of redundancy is in this case equal to 4 . 


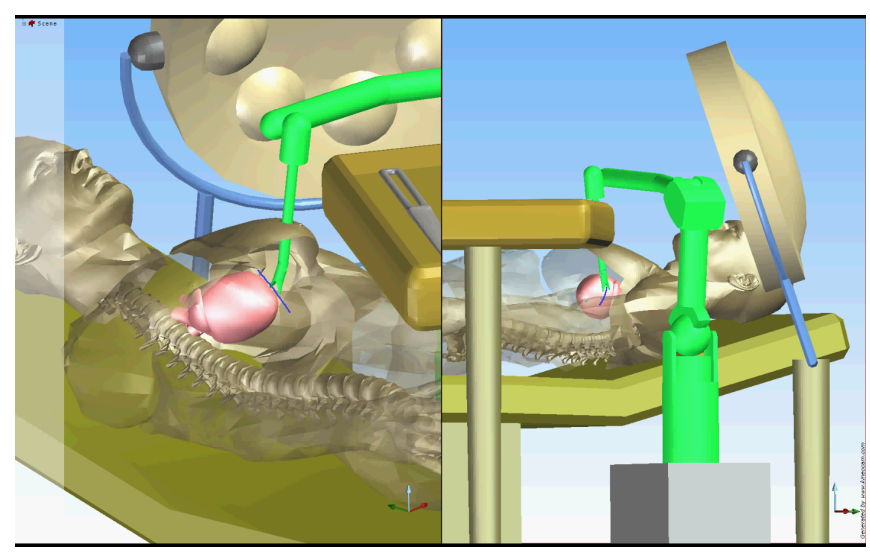

Fig. 8. Motion planning scenario. The manipulator must follow the assigned blue path near the hart while satisfying the RCM constraint and avoiding undesired contacts.

To solve the motion planning problem, we have applied the control-based task constrained motion planning scheme presented in [23] with the motion generation scheme given by the kinematic control law (7). This implies that the local paths used by the planner satisfy the RCM and the task path constraints. The redundant input $w$ has been used to avoid obstacles. Animation of the solution path is shown in the accompanying video.

\section{CONCLUSION AND FUTURE WORK}

We have proposed a general formalization of the RCM constraint arising in the context of minimally invasive robotic surgery. With respect to the literature, this model has the advantage of requiring minimal knowledge of the trocar geometry and of allowing direct control on the penetration of the surgical instrument inside the patient's body. For a generic task we have derived a constrained kinematic controller guaranteeing exponential convergence of the task with stable satisfaction of the RCM constraint. Application to a visual servoing task and a motion planning problem have been proposed to validate the approach.

It is well known that kinematic task controller like (7) can guarantee only local convergence in the presence of parameter uncertainties characterizing practical applications [20]. Additional stability and robustness analysis specific to the considered application would therefore be necessary like, e.g., in [15], [16]. The closure of a force control loop would also prove necessary to guarantee safe interaction. Finally, in the current control scheme the unintentional motion of the patient, like heart beating and breathing are not considered. In future work we will address all these issues with the aim of validating experimentally the proposed RCM constraint modeling for the execution of surgical tasks designed in collaboration with a surgeon.

\section{REFERENCES}

[1] R. H. Taylor, P. Kazanzides, and F. D. Dagan, Medical Robotics and Computer-Integrated Interventional Medicine. Biomedical Information Technology Burlington: Academic Press, 2008.
[2] R. H. Taylor and D. Stoianovici, "Medical robotics in computer integrated surgery," IEEE Trans. on Robotics and Automation, vol. 19, pp. 765-781, 2003.

[3] C.-H. Kuo and J. S. Dai, "Robotics for minimally invasive surgery: A historical review from the perspective of kinematics," in Int. Symp. on History of Machines and Mechanisms, 2009, pp. 337-354.

[4] U. H. et al., "Telemanipulator for remote minimally invasive surgery," IEEE Robotics \& Automation Magazine, vol. 15, pp. 28-38, 2008.

[5] M. Michelin, P. Poignet, and E. Dombre, "Dynamic task/posture decoupling for minimally invasive surgery motions," in 2004 IEEE/RSJ Int. Conf. on Intelligent Robots and Systems, 2004, pp. 3625-3630.

[6] H. Mayer, I. Nagy, and A. Knoll, "Kinematics and modeling of a system for robotic surgery," in Advances in Robot Kinematics, 2004.

[7] T. Ortmaier and G. Hirzinger, "Cartesian control issues for minimally invasive robot surgery," in 2000 IEEE/RSJ Int. Conf. on Intelligent Robots and Systems, 2000, pp. 565-571.

[8] R. Locke and R. Patel, "Optimal remote center-of-motion location for robotics-assisted minimally-invasive surgery," in 2007 IEEE Int. Conf. on Robotics and Automation, 2007, pp. 1900-1905.

[9] J. Funda, R. H. Taylor, B. Eldridge, S. Gomory, and K. G. Gruben, "Constrained cartesian motion control for teleoperated surgical robots," IEEE Trans. on Robotics and Automation, vol. 12, pp. 453-465, 1996.

[10] M. Li and R. Taylor, "Performance of surgical robots with automatically generated spatial virtual fixtures," in 2005 IEEE Int. Conf. on Robotics and Automation, 2005, pp. 217-222.

[11] M. Li, A. Kapoor, and R. Taylor, "A constrained optimization approach to virtual fixtures," in IEEE/RSJ Int. Conf. on Intelligent Robots and Systems, 2005, pp. 1408-1413.

[12] M. Michelin, P. Poignet, and E. Dombre, "Geometrical control approaches for minimally invasive surgery," in Medical Robotics, Navigation and Visualization (MRNV'04), 2004.

[13] H. Azimian, R. Patel, and M. Naish, "On constrained manipulation in robotics-assisted minimally invasive surgery," in $20103 \mathrm{rd}$ IEEE RAS and EMBS Int. Conf. on Biomedical Robotics and Biomechatronics, 2010, pp. 650-655.

[14] R. Ginhoux, J. Gangloff, M. de Mathelin, L. Soler, M. M. A. Sanchez, and J. Marescaux, "Active filtering of physiological motion in robotized surgery using predictive control," IEEE Trans. on Robotics, vol. 21 , pp. 67-79, 2005

[15] A. Krupa, J. Gangloff, C. Doignon, M. de Mathelin, G. Morel, J. Leroy, L. Soler, and J. Marescaux, "Autonomous 3-d positioning of surgical instruments in robotized laparoscopic surgery using visual servoing," IEEE Trans. On Robotics and Automation, vol. 19, pp. 842852, 2003.

[16] M.-A. Vitrani, H. Mitterhofer, N. Bonnet, and G. Morel, "Robust ultrasound-based visual servoing for beating heart intracardiac surgery," in 2007 IEEE Int. Conf. on Robotics and Automation, 2007, pp. 3021-3027.

[17] A. J. W. P. Hynes, G. I. Dodds, "Uncalibrated visual-servoing of a dual-arm robot for mis suturing," in The First IEEE/RAS and EMBS Int. Conf. on Biomedical Robotics and Biomechatronics, 2006, pp. $420-425$.

[18] C. S. T. Osa and A. Knoll, "Framework of automatic robot surgery system using visual servoing," in 2010 IEEE/RSJ Int. Conf. on Intelligent Robots and Systems, 2010, pp. 1837-1842.

[19] F. Bourger, C. Doignon, P. Zanne, and M. de Mathelin, "A modelfree vision-based robot control for minimally invasive surgery using esm tracking and pixels color selection," in 2007 IEEE Int. Conf. on Robotics and Automation, 2007, pp. 3579 -3584.

[20] F. Chaumette and S. Hutchinson, "Visual servo control, part i: Basic approaches," IEEE Robotics and Automation Mag., vol. 13, pp. 82-90, 2006.

[21] A. De Luca, G. Oriolo, and P. Robuffo Giordano, "Feature depth observation for image-based visual servoing: Theory and experiments," The International Journal of Robotics Research, vol. 27, no. 10, pp. 1093-1116, 2008.

[22] S. L. Chiu, "Task compatibility of manipulator postures," The International Journal of Robotics Research, vol. 7, pp. 13-21, 1988.

[23] G. Oriolo and M. Vendittelli, "A control-based approach to task- constrained motion planning," in 2009 IEEE/RSJ Int. Conf. on Intelligent Robots and Systems, 2009, p. 297302. 